\title{
Impacto de la norma social subjetiva en la intención de emprendimiento sostenible: un caso de estudio con estudiantes colombianos
}

DOI: https://doi.org/10.21158/01208160.n85.2018.2048

Fecha de recepción: 13 de febrero de 2018

Fecha de aprobación: 25 de junio de 2018

Edgar Julián Gálvez-Albarracín ${ }^{1}$ Universidad del Valle edgar.galvez@correounivalle.edu.co

Rafael Andrés Guauña-Aguilar ${ }^{2}$ Cámara de Comercio del Cauca formacionstder@cccauca.org.co

Rafael Ignacio Pérez-Uribe ${ }^{3}$ Universidad EAN riperez@universidadean.edu.co

Cómo citar este artículo / To reference this article / Comment citer cet article / Para citar este artigo:

Gálvez-Albarracín, E. J.; Guauña-Aguilar, R. A. y Pérez-Uribe, R. I. (2018). Impacto de la norma social subjetiva en la intención de emprendimiento sostenible: un caso de estudio con estudiantes colombianos. Revista EAN, 85, 57-74. DOI: https://doi. org/10.21158/01208160.n85.2018.2048

\section{Resumen}

El objetivo de este artículo, basado en la investigación científica que lo sustenta, es aportar nuevas evidencias en el campo del emprendimiento relacionado con sostenibilidad, las intenciones emprendedoras y la creación de empresas en estudiantes universitarios, en el Valle del Cauca. Se trabajó con una muestra de 246 estudiantes de contaduría pública y administración de empresas de la Universidad del Valle, Sede Norte del Cauca. Los resultados señalan que el 70 \% de alumnos estaban interesados en ser empresarios con enfoque sostenible, siendo la variable con mejor valoración el apoyo de la familia y que a mejor percepción de la norma subjetiva mayor intención emprendedora sostenible en las personas. De esta manera se comprobó la hipótesis formulada: cuanto mayor es en los estudiantes la percepción de una positiva valoración social respecto a la creación de empresas sostenibles, mayor es su actitud emprendedora.

\section{Palabras clave}

Estudiantes universitarios - Valle del Cauca (Colombia)-, emprendimiento, sostenibilidad, creación de empresas, desarrollo sostenible.

\footnotetext{
${ }^{1}$ Doctor en Administración y Dirección de Empresas, Universidad Politécnica de Cartagena, España. Magíster en Creación de Empresas y Gestión de Proyectos de Innovación, Universidad Bordeaux IV, Francia. Administrador de Empresas, Universidad del Valle, Colombia. ORCID: https://orcid.org/0000-0003-3972-2310

${ }^{2}$ Magister en Administración de Empresas de la Universidad del Valle. Coordinador de Fortalecimiento Empresarial de la Cámara de Comercio del Cauca.

${ }^{3}$ Doctor en Ciencias Empresariales Universidad Nebrija. Diploma de Estudios Avanzados en Economía Aplicada, Universidad Nebrija. Maestría en Gestión de Organizaciones Universidad EAN. Maître es Sciences Université du Québec a Chicoutimi. Especialista en Evaluación y Construcción de Indicadores de Gestión, Escuela de Administración de Negocios. Estudios de Postgrado como Kenkyusei (Investigador adscrito) en Control Total de la Calidad y Círculos de Calidad, Fukushima University, Japón. Administrador de Empresas, Universidad Jorge Tadeo Lozano. ORCID: https://orcid.org/0000-00019924-6657
} 


\title{
Impact of subjective social norm in an intent to sustainable entrepreneurship: a case study with Colombian students
}

\begin{abstract}
This scientific research article aims at showing evidences of sustainable entrepreneurship, entrepreneurial intents and company creation of university students in Valle del Cauca. This study involve 246 accounting and business administration students from Universidad del Valle, in its northern site in Cauca. The outcomes show that $70 \%$ of the students are interested in becoming managers with a sustainable perspective, being the variable with the best family perception. Also, it demonstrates that having a better perception of the subjective norm causes a major sustainable entrepreneurial intent in students. Therefore, the formulated hypothesis was proved: the bigger the perception of a social positive valuation about the creation of sustainable companies, the better their entrepreneurial spirit.
\end{abstract}

Key words. University students- Valle del Cauca (Colombia), entrepreneurship, creating companies, sustainable development.

\section{Impact de la norme sociale subjective sur les intentions de l'entrepreneuriat durable: une étude de cas avec des étudiants colombiens}

Résumé. L'objectif de cet article scientifique est de fournir de nouvelles évidences en rapport à l'entrepreneuriat lié au développement durable, aux intentions entrepreneuriales et à la création d'entreprises par des étudiants universitaires de la Valle del Cauca. L'étude porte sur un échantillon de 246 étudiants de comptabilité publique et gestion des affaires de l'Universitédu Valle (Cauca Norte). Les résultats indiquent que $70 \%$ des étudiants souhaitent devenir entrepreneurs militant pour une approche durable de leurs activités et montrent que la variable ayant le plus de valeur aux yeux des étudiants reste le soutien famillial dans la mesure où une meilleure perception de cette norme subjective stimule une plus grande intention entrepreneuriale eco-reponsable des participants. L'hypothèse de départ a pu être prouvée: plus les étudiants ont une perception positive d'une évaluation sociale positive de création d'entreprises eco-responsables, plus leur attitude entrepreneuriale est élevée.

Mots clefs. Étudiants universitaires - Valle del Cauca (Colombie), esprit d'entreprise, durabilité, création d'entreprises, développement durable.

\section{Impacto da norma social subjetiva na intenção de empreendimento sustentável: um estudo de caso com estudantes colombianos}

Resumo. O objetivo deste artigo, baseado na pesquisa científica que o sustenta, é contribuir com novas evidência no campo do empreendimento relacionado com a sustentabilidade, com as intenções empreendedoras e com a criação de empresas em estudantes universitários, no Valle del Cauca (Colômbia). Trabalhou-se com uma mostra de 246 estudantes de contabilidade pública e administração de empresas da Universidade del Valle, Sede Norte do Cauca. Os resultados indicam que $70 \%$ dos alunos estavam interessados em ser empresários com enfoque sustentável, sendo a variável com melhor valorização o apoio da família e quanto melhor a percepção da norma subjetiva, maior a intenção empreendedora sustentável nas pessoas. Desta maneira comprovou-se a hipótese formulada: quanto maior a percepção de uma positiva valorização social com respeito à criação de empresas sustentáveis nos estudantes, maior é sua atitude empreendedora.

Palavras-chave. Estudantes universitários - Valle del Cauca (Colômbia), empreendimento; sustentabilidade, criação de empresas, desenvolvimento sustentável. 


\section{Introducción}

$\mathrm{E}^{1}$ emprendimiento sostenible y su manifestación en la creación de empresas son considerados dinamizadores del desarrollo social y económico de las naciones y las regiones (Saravia, 2010), razón por la cual el sector público, el privado y la academia muestran interés creciente por entender dichos fenómenos y los elementos que los estimulan y favorecen (Hernández et al., 2017; Varela, 2008; Audretsch, Belitski y Desai, 2007), por lo que busca que cada vez más estudiantes y profesionales universitarios se conviertan en empresarios con amplia visión de sostenibilidad de sus empresas (García, Mendoza y Romo, 2017; Goyanes, 2015; Bae, Qian, Miao y Fiet, 2014; Joensuu, Varamäki y Viljamaa, 2015; González, 2004).

En dicho contexto, es importante tener en cuenta que en cualquier proceso de emprendimiento hacia la sostenibilidad, intervienen las personas y los entornos general y empresarial en los que debe encontrar oportunidades y recursos, y que para autores como Vainrub (2009) y Timmons y Spinelli (2004) el principal es el humano, por lo que su potencial emprendedor es estudiado desde enfoques como sus aptitudes (Mora, 2011) y perfiles sociales y psicosociales (Novillo, Sarmiento, Ollague y Ramón, 2017; Moriano, Trejo y Palaci, 2001).

De acuerdo al modelo conceptual del Global Entrepreneurship Monitor (GEM), estudio que mide la tasa de actividad emprendedora (TEA) en diferentes países y regiones, una de las fases en la creación de empresas es la de intención, en la cual a los individuos que están considerando hacerlo se les define como empresarios intencionales. En ese sentido, numerosas investigaciones como las de Laguía,
Moriano, Molero y Gámez (2017); Rueda, Fernández Herrero (2012); y Sánchez, Lanero y Yurrebaso (2005) han confirmado que la intención es una buena variable predictora del inicio de empresas.

No obstante a lo señalado, el informe GEM 2016 muestra para Colombia que mientras un $53 \%$ de las personas encuestadas en este país eran empresarios intencionales, solo el 16.3 $\%$ habían iniciado efectivamente actividades empresariales (Buelvas et al., 2017), habiéndose desaprovechado en consecuencia o fugado por la tubería empresarial el restante $26.7 \%$ de las intenciones de crear nuevas empresas, situación que debe interesar y preocupar a la clase dirigente e intelectual.

En ese sentido, a nivel mundial numerosos trabajos como los de Hernández et al. (2017) y Valencia, Montoya y Montoya (2015), han confirmado la utilidad de los conceptos aportados por la Teoría del Comportamiento Planificado desarrollada por Ajzen en 1991, para identificar y describir los factores que estimulan o desalientan en los individuos de diferentes condiciones y características la intención de crear empresas, por lo cual en este documento se le denominará como intención emprendedora; sin embargo, dichos estudios se han aplicado principalmente en las grandes ciudades, sin profundizar en las más pequeñas que generalmente difieren en su ecosistema empresarial respecto a las primeras.

En ese escenario, la Universidad del Valle, sede Norte del Cauca, ubicada en Colombia en el municipio de Santander de Quilichao, atiende a su población y a la de otros 13 municipios de tamaño igualmente pequeño en los departamentos del Cauca y

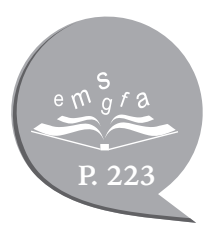


del sur del Valle del Cauca, los cuales son importantes para la región y el país por su potencial económico y su problemática social.

De acuerdo con lo señalado anteriormente en este trabajo de investigación, que contribuye a llenar el vacío de literatura descrito en relación con el emprendimiento sostenible universitario en ciudades no capitales, se responde a las preguntas: ¿Cuál es el nivel de interés por crear sus propias empresas sostenibles por parte de los estudiantes de Contaduría Pública y Administración de Empresas de la Universidad del Valle, sede Norte del Cauca? ¿Cómo la idea de la forma en que la sociedad percibe dicha actividad influye positiva o negativamente en ello?

El presente artículo está estructurado de tal manera que en la primera parte se desarrolla el contenido del trabajo, lo que incluye el marco teórico, los estudios previos encontrados, las hipótesis de investigación, la metodología utilizada y se presentan y discuten los resultados y en la segunda se plantean sus conclusiones, limitaciones y futuras líneas de trabajo.

\section{Marco teórico}

\begin{abstract}
A continuación, se describen los princi$\Lambda_{\text {pales aspectos teórico-conceptuales que }}$ enmarcan la presente investigación:
\end{abstract}

\subsection{Emprendimiento, emprendedor e intención emprendedora}

El emprendimiento y los emprendedores han sido objeto de estudio para importantes autores (Diez, 2016) debido a su relevante papel en el cambio y el desarrollo socioeconómico, es así que Schumpeter (1950) los liga al concepto de empresario innovador (RamirezSalazar, Perez-Uribe y Salcedo-Perez, 2018), entendiéndolo como aquel que es capaz de romper la inercia de los mercados para renovarlos de manera permanente.

El término emprendimiento, que en francés se denomina entrepreneuriat y en inglés entrepreneurship, es definido en el Diccionario de la Real Academia de la Lengua Española (DRAE) como una de sus acepciones «acción de emprender», al emprender como "acometer y comenzar una obra, un negocio, un empeño, especialmente si encierran dificultad o peligro", $\mathrm{y}$ al emprendedor como quien «emprende con resolución acciones o empresas innovadoras» (RAE, 2017).

Diferentes autores se refieren también al emprendimiento con otros términos, como actividad emprendedora o espíritu empresarial, y señalan que tienen tres elementos fundamentales: a) el individuo o grupo de individuos que lo ejercen, b) el medio o entorno en el cual se desarrolla, y c) la acción o actividad emprendedora como tal; es por ello que para entenderlo como fenómeno económico y social ha sido estudiado desde diferentes enfoques o corrientes, entre ellas la económica, la sociopsicológica y la comportamental (Gómez, 2010).

Según Alonso (2012) en relación con la creación de empresas, ya en 1984 Shapero señalaba que esta podía estar animada o desestimulada por las percepciones de deseabilidad y de factibilidad que el emprendedor tuviera de dicha acción. Posteriormente autores como Krueger (2000) y Liñan (2004), señalaron además de la importancia de los rasgos de 
personalidad y los factores demográficos, agregar a dicho análisis elementos como la intención, lo cual podría hacerse a través de teorías como la del comportamiento planificado de Ajzen (1991).

\subsection{Emprendimiento y sostenibilidad}

Cortés y Peña (2015, p. 43) ofrecen la siguiente definición, que aclara de tajo el concepto de lo sostenible:

En la literatura en español, se encuentra la utilización de los términos sostenible y sustentable; desarrollo sostenible y desarrollo sustentable; sostenibilidad y sustentabilidad. Sin embargo, quienes utilizan las expresiones se fundamentan en el término sustainability, como referente para sus publicaciones. Así por ejemplo, se pueden encontrar artículos científicos que traducen sustainability como sostenibilidad, y a su vez, otros artículos que traducen el término como sustentabilidad, incluso cuando la referencia citada es la misma (p. 53).

Rodríguez (2016), citando a Schaltegger y Wagner (2011), afirma que emprendimiento sostenible es la realización de una innovación dirigida a un segmento de mercado que proporciona beneficio a gran parte de la sociedad. Desde el punto de vista de la gestión ambiental las organizaciones que hacen de esta dimensión su actividad principal se pueden llamar empresas sostenibles, generando nuevos productos bienes y servicios-, herramientas y métodos de organización que permitan trabajar en armonía con el ecosistema y aumenten la calidad de vida. Este enfoque implica lograr el desarrollo sostenible, mediante el descubrimiento, evaluación y explotación de oportunidades y la creación de valor para generar prosperidad económica, cohesión social y reconstrucción y protección del medio ambiente.

\subsection{Teoría del comportamiento planificado - TCP}

La TCP utilizada en múltiples investigaciones para medir la intención emprendedora de las personas (Valencia, Montoya y Montoya, 2016), fue propuesta por el psicólogo social Icek Ajzen en 1991 como complemento de la teoría de la acción razonada (Ajzen y Fishben, 1980) buscando perfeccionar el marco de comprensión de los antecedentes de las acciones de los individuos. La TCP que ha derivado en diversos cuestionarios como los utilizados por Liñán, Moriano y Jaén (2016), postula como predictores de la creación de empresas los siguientes factores: la actitud hacia el comportamiento, la norma subjetiva y el control percibido o autoeficacia. La figura 1 muestra el modelo conceptual propuesto: 
Figura 1. Teoría del Comportamiento Planificado (TCP)

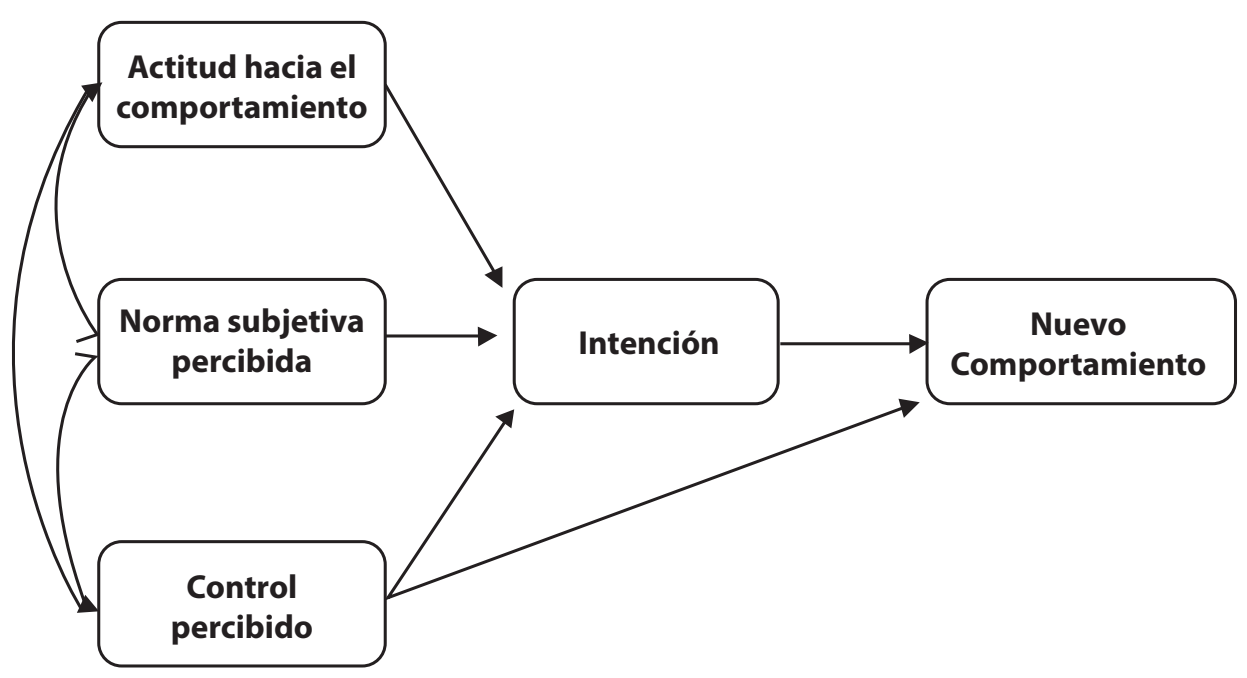

Fuente. Adaptado de Ajzen, 1991.

Finalmente, el control percibido o autoeficacia lo define como la percepción o grado de confianza que tiene el individuo en relación con su capacidad y preparación para realizar una determinada actividad.

El segundo factor de la teoría de Ajzen (1991) es en el que profundiza esta investigación, tanto para conocer la valoración de la percepción que tiene la sociedad sobre la creación de empresas, como el impacto que ello tiene en su intención emprendedora. A continuación, se relacionan trabajos encontrados en relación con la teoría del Comportamiento Planificado, la norma subjetiva y la intención emprendedora, mismos que aportan elementos conceptuales o empíricos a la presente investigación (Tabla 1). 
Tabla 1. Estudios representativos

\begin{tabular}{|c|c|c|}
\hline Autores & Estudio & Hallazgos \\
\hline $\begin{array}{l}\text { Sánchez, Lanero y } \\
\text { Yurrebaso (2005). }\end{array}$ & $\begin{array}{l}\text { Buscan desarrollar un modelo } \\
\text { psicológico integrador sobre } \\
\text { la formación de intenciones } \\
\text { emprendedoras, analizan sus variables } \\
\text { determinantes en la Universidad de } \\
\text { Salamanca, España. }\end{array}$ & $\begin{array}{l}\text { De los factores frecuentemente } \\
\text { estudiados los más importantes allí } \\
\text { fueron la autoeficacia, la propensión al } \\
\text { riesgo y la proactividad. }\end{array}$ \\
\hline $\begin{array}{l}\text { Martínez y Campos } \\
\text { (2011). }\end{array}$ & $\begin{array}{l}\text { Con una muestra de estudiantes de la } \\
\text { Universidad de Deusto (España). }\end{array}$ & $\begin{array}{l}\text { Encuentran que la mayor parte de } \\
\text { alumnos creen que sus amigos, } \\
\text { compañeros y familiares les apoyarían } \\
\text { en su proceso emprendedor, siendo la } \\
\text { opinión de sus familias especialmente } \\
\text { importante para ellos. }\end{array}$ \\
\hline $\begin{array}{l}\text { Rifelly y Martdianty } \\
\text { (2012). }\end{array}$ & $\begin{array}{l}\text { Pusieron a prueba la TCP para con una } \\
\text { muestra de } 600 \text { estudiantes explicar } \\
\text { la intención de iniciar empresa en seis } \\
\text { universidades de Indonesia. }\end{array}$ & $\begin{array}{l}\text { Sus resultados mostraron que dicha } \\
\text { teoría fue útil para predecir la intención } \\
\text { emprendedora simultáneamente en las } \\
\text { seis universidades y en el total de los } \\
\text { alumnos observados. }\end{array}$ \\
\hline Yang (2013). & $\begin{array}{l}\text { Con una muestra de } 1.330 \text { estudiantes } \\
\text { chinos de la Universidad de Finanzas y } \\
\text { Economía Jiangxi. }\end{array}$ & $\begin{array}{l}\text { Encontró que la norma subjetiva, es } \\
\text { un buen predictor de la intención } \\
\text { emprendedora. }\end{array}$ \\
\hline $\begin{array}{l}\text { Rueda, Sánchez, } \\
\text { Blanco y Fernández } \\
\text { (2013). }\end{array}$ & $\begin{array}{l}\text { Publican los resultados de una } \\
\text { investigación realizada con } 525 \\
\text { alumnos de la Universidad de } \\
\text { Cantabria, España, en la cual utilizando } \\
\text { la TCP buscan conocer los factores } \\
\text { que motivan o frenan su intención } \\
\text { emprendedora. }\end{array}$ & $\begin{array}{l}\text { Entre sus resultados encuentran que en } \\
\text { cuanto a las motivaciones las mujeres le } \\
\text { dan más importancia que los hombres } \\
\text { a las recompensas personales y menos } \\
\text { que ellos a las recompensas económicas. } \\
\text { Además que están relacionados con las } \\
\text { habilidades y capacidades del individuo } \\
\text { para desarrollar un proyecto empresarial, } \\
\text { con la influencia social percibida en } \\
\text { su entorno próximo, así como con sus } \\
\text { creencias. }\end{array}$ \\
\hline $\begin{array}{l}\text { Chan, Escalante y } \\
\text { Robles (2015). }\end{array}$ & $\begin{array}{l}\text { Un estudio en el que comparan } \\
\text { la intención emprendedora en } \\
\text { estudiantes de carreras económicas y } \\
\text { administrativas en dos universidades } \\
\text { ubicadas en Puerto Rico y México. }\end{array}$ & $\begin{array}{l}\text { Sus hallazgos muestran que los factores } \\
\text { que más favorecen el querer crear empresa } \\
\text { son el buen manejo de problemas, la } \\
\text { creatividad, la seguridad y la aceptación } \\
\text { del riesgo, siendo este último factor el } \\
\text { que marca la diferencia entre los alumnos } \\
\text { de los dos países. }\end{array}$ \\
\hline $\begin{array}{l}\text { Vargas y Bedoya } \\
(2015) \text {. }\end{array}$ & $\begin{array}{l}\text { Publican su trabajo de grado de } \\
\text { Maestría en Administración de } \\
\text { Empresas en el que a través de la TCP } \\
\text { analizaron los factores psicológicos que } \\
\text { influyen en la intención emprendedora } \\
\text { de } 292 \text { estudiantes de nivel tecnológico } \\
\text { del Servicio Nacional de Aprendizaje de } \\
\text { Colombia (SENA). }\end{array}$ & $\begin{array}{l}\text { Sus resultados muestran que el } 71 \% \\
\text { de los estudiantes manifestaron que } \\
\text { era probable o totalmente probable que } \\
\text { llegaran a crear empresa, siendo dicha } \\
\text { intención más alta en los de contabilidad } \\
\text { y finanzas que en los de procesos } \\
\text { administrativos. }\end{array}$ \\
\hline
\end{tabular}


Tabla 1. Estudios representativos (Continuación)

\begin{tabular}{|c|c|c|}
\hline $\begin{array}{l}\text { Marín, Rubio y } \\
\text { Sánchez (2015). }\end{array}$ & $\begin{array}{l}\text { Publican los resultados de un estudio } \\
\text { realizado con } 335 \text { alumnos de la } \\
\text { Universidad de Murcia, España en } \\
\text { el que analizan los determinantes } \\
\text { de la intención emprendedora de los } \\
\text { estudiantes universitarios. }\end{array}$ & $\begin{array}{l}\text { Concluyen que si las universidades, } \\
\text { aumentan el número de estudiantes con } \\
\text { una actitud positiva hacia la creación de } \\
\text { empresas, lograrán a futuro tener muchos } \\
\text { más que consideren la vida empresarial } \\
\text { como una valiosa opción de desarrollo } \\
\text { profesional. }\end{array}$ \\
\hline Guañua (2015). & $\begin{array}{l}\text { Con una muestra de } 246 \text { estudiantes } \\
\text { de la Facultad de Ciencias de la } \\
\text { Administración de la Universidad del } \\
\text { Valle en Colombia. }\end{array}$ & $\begin{array}{l}\text { Encuentra en su trabajo de grado de } \\
\text { Maestría, que la valoración positiva de } \\
\text { la norma subjetiva impulsa la intención } \\
\text { emprendedora de los estudiantes de } \\
\text { ciudades pequeñas y no capitales. }\end{array}$ \\
\hline Fernández (2017). & $\begin{array}{l}\text { Publica los resultados de su trabajo } \\
\text { de grado para optar por su título en } \\
\text { la Facultad de Turismo y Finanzas de } \\
\text { la Universidad de Sevilla, España, en } \\
\text { la cual a través de la TCP y con una } \\
\text { muestra de } 204 \text { alumnos pretendía } \\
\text { encontrar las diferencias entre la } \\
\text { intención emprendedora entre hombres } \\
\text { y mujeres. }\end{array}$ & $\begin{array}{l}\text { Sus resultados muestran que los alumnos } \\
\text { del género masculino tienen mayor } \\
\text { intención que las del femenino lo cual } \\
\text { intentan explicar en la percepción que las } \\
\text { mismas mujeres tienen de las ocupaciones } \\
\text { que deben asumir en la sociedad como } \\
\text { ser amas de casa, y en el tener mayor } \\
\text { dificultad para acceder a financiamiento } \\
\text { bancario. }\end{array}$ \\
\hline $\begin{array}{l}\text { Jaimes, Jaramillo y } \\
\text { Pérez (2017). }\end{array}$ & $\begin{array}{l}\text { Publican los resultados de una } \\
\text { investigación en la cual buscan } \\
\text { identificar los factores que inciden } \\
\text { en la intención emprendedora } \\
\text { de los estudiantes de las áreas } \\
\text { económico-administrativas del Centro } \\
\text { Universitario Temascaltepec de la } \\
\text { Universidad Autónoma del Estado de } \\
\text { México. Para ello toman una muestra } \\
\text { de } 342 \text { estudiantes de las licenciaturas } \\
\text { en Administración, Contaduría e } \\
\text { Informática Administrativa. }\end{array}$ & $\begin{array}{l}\text { Los resultados revelan que los factores } \\
\text { sociodemográficos que inciden en } \\
\text { la intención emprendedora de los } \\
\text { estudiantes son su semestre, estado civil, } \\
\text { edad y conocimiento del programa de } \\
\text { emprendimiento; los factores psicológicos } \\
\text { más influyentes son propensión al } \\
\text { riesgo, autoestima e innovación, y el } \\
\text { socioeconómico es la tendencia a la } \\
\text { inversión. }\end{array}$ \\
\hline $\begin{array}{l}\text { Hernández et al. } \\
\text { (2017). }\end{array}$ & $\begin{array}{l}\text { Publican los resultados del } \\
\text { informe GUESSS (Global University } \\
\text { Entrepreneurial Students' Survey) donde } \\
\text { entre otros factores y también mediante } \\
\text { la TCP miden en una muestra } 2.497 \\
\text { estudiantes la intención emprendedora } \\
\text { en la Universidad de Extremadura en } \\
\text { España. }\end{array}$ & $\begin{array}{l}\text { Encuentran que en una escala de } 1 \text { a } \\
7 \text { donde } 1 \text { es nada dispuestos a crear } \\
\text { empresa y } 7 \text { totalmente, la media del } \\
\text { total de encuestados estuvo alrededor } \\
\text { de } 4 \text { lo que está por encima del valor } \\
\text { medio -3.5-, sin embargo también hallan } \\
\text { que solo el } 4.7 \% \text { de los estudiantes } \\
\text { quisieran implicarse en crear empresas } \\
\text { inmediatamente después de terminar } \\
\text { sus estudios, cifra que sube al } 29.9 \% \\
\text { a los } 5 \text { años de haberlo hecho. Por otra } \\
\text { parte, encuentran que la valoración } \\
\text { social positiva incrementa la intención } \\
\text { emprendedora de los encuestados. }\end{array}$ \\
\hline
\end{tabular}


Tabla 1. Estudios representativos (Continuación)

\begin{tabular}{|c|c|c|}
\hline $\begin{array}{l}\text { González, Torres y } \\
\text { Tinoco (2017). }\end{array}$ & $\begin{array}{l}\text { Publican los resultados de su } \\
\text { investigación en la cual con una } \\
\text { muestra de } 618 \text { de estudiantes de } \\
\text { licenciatura de la Universidad de } \\
\text { Colima en México. }\end{array}$ & $\begin{array}{l}\text { Encuentran que mediante modelos } \\
\text { de regresión multinomiales que una } \\
\text { situación socioeconómica privilegiada, } \\
\text { la experiencia familiar en los negocios, } \\
\text { la edad y una alta percepción de espíritu } \\
\text { emprendedor y capacidad de trabajo son } \\
\text { factores que aumentan la probabilidad } \\
\text { de que el estudiante tenga una alta } \\
\text { experiencia emprendedora. }\end{array}$ \\
\hline
\end{tabular}

Fuente. Elaboración propia.

\section{Hipótesis de investigación}

T eniendo en cuenta el problema de investigación planteado, el marco teóricoconceptual y los estudios previos señalados, se plantea las siguientes hipótesis de investigación:
H: cuanto mayor es en los estudiantes la percepción de una positiva valoración social respecto a la creación de empresas sostenibles, mayor es su actitud emprendedora.

\section{Metodología de la investigación}

Gte estudio es de corte transversal, descriptivo y explicativo, ya que busca observar y analizar para un momento específico los factores que influyen positiva o negativamente sobre la intención emprendedora de los estudiantes objetivo, es decir los 533 matriculados durante el periodo académico febrero a junio del 2015 en las carreras profesionales de Administración de Empresas y Contaduría Pública de la Universidad del Valle, sede Norte del Cauca; programas en los que se incluyen las asignaturas de creación de empresas y electivas en áreas de profundización en emprendimiento sostenible.

Para determinar el tamaño de la muestra se utilizó la fórmula de Jany (2009) para poblaciones finitas, la cual con un margen de confianza del $95 \%$ y de error del $5 \%$ indicó que debía ser como mínimo de 223 individuos. El muestreo fue aleatorio y estratificado con asignación proporcional, tomando como variables el programa académico -Administración de Empresas y Contaduría Pública-, el género de los alumnos, la jornada-diurna o nocturna-, el ciclo académico -fundamentación o profesional-y el estrato socioeconómico. La recolección de datos se realizó personalmente en junio de 2015 a través de la aplicación de un cuestionario estructurado, basado en los utilizados de manera internacional (Pérez, Zárate, Díaz, Martínez y Vargas, 2015; Laguía et al., 2017). El procesamiento de los datos se realizó utilizando técnicas y herramientas de estadística descriptiva e inferencial según fuera el objetivo abordado. 
La tabla 2 permite ver que la muestra quedó constituida por 246 estudiantes, de los cuales 73 eran hombres -30\%-y 173 mujeres -70 $\%-$, que 78 estaban en la jornada diurna $-31 \%-$ y 168 en la nocturna $-69 \%-, 118$ cursaban el ciclo profesional -48 \%-y 128 el de fundamentación $-52 \%-$; y finalmente que 243 eran de estrato socioeconómico 1-3 -99 \%- y el solo 3 de los estratos 4-6 -1\%-.

Tabla 2. Configuración de la muestra

\begin{tabular}{|c|c|c|c|c|c|c|c|c|c|}
\hline & \multicolumn{2}{|c|}{ Género } & \multicolumn{2}{c|}{ Ciclo } & \multicolumn{2}{c|}{ Jornada } & \multicolumn{2}{c|}{ Estrato } & \\
\hline Programa & M & H & Prof & Fund & Noc & Diu & $4-6$ & $1-3$ & Total \\
\hline Administración & 97 & 43 & 51 & 89 & 85 & 55 & 3 & 137 & 140 \\
\hline Contaduría & 76 & 30 & 67 & 39 & 83 & 23 & 0 & 106 & 106 \\
\hline Totales & 173 & 73 & 118 & 128 & 168 & 78 & 3 & 243 & 246 \\
\hline
\end{tabular}

Fuente. Elaboración propia.

\subsection{Medición de variables y validación de escalas.}

A continuación se muestran las variables que fueron observadas y la forma en que se midió cada una, puede verse que en todos los casos se utilizaron escalas tipo Likert.

\subsubsection{Intención emprendedora o creación} de empresa sostenible propia.

Para medir esta variable se preguntó a los estudiantes cuál era su disposición actual para crear una empresa sostenible propia, ofreciéndoles las siguientes alternativas: 1) Nada dispuesto; 2) Poco dispuesto; 3) Medianamente dispuesto; 4) Dispuesto y; 5) Totalmente dispuesto.

\subsubsection{Valoración social de la creación de empresas.}

La tabla 3 muestra las preguntas que se formularon y las opciones que se utilizaron para conocer la percepción que tenían los estudiantes sobre la valoración social de la creación de empresas sostenibles, puede verse en el estadístico Alpha de Cronbach de 0,70 -cercano a 1-que la escala es fiable, es decir que mide adecuadamente la variable revisada (Mercado y Gil, 2012, p. 266). 
Tabla 3. Valoración social de la creación de empresas sostenibles

Señale su grado de acuerdo o de desacuerdo con las siguientes afirmaciones, teniendo en cuenta la siguiente escala: 1. Totalmente en desacuerdo, 2. En desacuerdo, 3. Ni de acuerdo ni en desacuerdo, 4. De acuerdo y 5. Totalmente de acuerdo:

La cultura general de mi país es muy favorable hacia la actividad de los empresarios con actitud de sostenibilidad.

Mi familia directa valora positivamente la actividad en términos de sostenibilidad de los empresarios.

Mis amigos valoran la actividad emprendedora sostenible por encima de otras profesiones.

En mi región se considera que la vocación emprendedora sostenible merece la pena.

Mis compañeros valoran la actividad emprendedora sostenible por encima de otras profesiones.

Fuente. Elaboración propia utilizando IBM SPSS Statistics 24.0.

\section{Resultados y discusión}

\subsection{Resultados descriptivos}

La tabla 4 muestra el nivel de intención emprendedora sostenible de los estudiantes abordados según las opciones planteadas. Se observa que del total un $69.92 \%$ estaba dispuesto o totalmente dispuesto a crear su propia empresa sostenible, un $20.73 \%$ medianamente y solo el $9.35 \%$ tenía ninguna o poca disposición a ello. Así mismo que la intención emprendedora sostenible era ligeramente más alta en la carrera de Contaduría Pública $(70.75 \%)$ que en la de Administración de Empresas (69.29%).

Tabla 4. Nivel de la intención emprendedora

\begin{tabular}{|c|c|c|c|c|c|c|c|c|c|c|c|c|}
\hline & \multicolumn{2}{|c|}{$\begin{array}{c}1 . \\
\text { Nada } \\
\text { dispuesto }\end{array}$} & $\begin{array}{c}2 . \\
\text { Poco } \\
\text { dispuesto }\end{array}$ & $\begin{array}{c}\text { 3. } \\
\text { Medianamente } \\
\text { dispuesto }\end{array}$ & \multicolumn{2}{|c|}{$\begin{array}{c}4 . \\
\text { Dispuesto }\end{array}$} & \multicolumn{2}{c|}{$\begin{array}{c}5 . \\
\text { Totalmente } \\
\text { dispuesto }\end{array}$} & \multicolumn{2}{c|}{ Totales } \\
\hline & Q & $\%$ & Q & $\%$ & Q & $\%$ & Q & $\%$ & Q & $\%$ & Q & $\%$ \\
\hline $\begin{array}{c}\text { En } \\
\text { Administración }\end{array}$ & 4 & 2.86 & 8 & 5.71 & 31 & 22.14 & 54 & 38.57 & 43 & 30.72 & 140 & 57 \\
\hline En Contaduría & 6 & 5.66 & 5 & 4.72 & 20 & 18.87 & 43 & 40.57 & 32 & 30.18 & 106 & 43 \\
\hline En el total & 10 & 4.07 & 13 & 5.28 & 51 & 20.73 & 97 & 39.43 & 75 & 30.49 & 246 & 100 \\
\hline
\end{tabular}

Fuente. Elaboración propia utilizando IBM SPSS Statistics 24.0. 
Los resultados señalados coinciden con los de Vargas y Bedoya (2015) respecto a que la mayor parte de la población estudiada estaba totalmente dispuesta, o medianamente dispuesta en emprender de manera sostenible, tan solo una minoría estaba poco o nada dispuesta. Con respecto a la intención empresarial fue más alta en los alumnos de áreas contables que en las administrativas.
Por su parte, la tabla 5 indica que la media de la intención emprendedora en el total de alumnos encuestados es de 3.87 , al acercarse al 4.0 señala que en general tienen buena disposición a crear empresa propia. Estos resultados coinciden con otros estudios realizados también en el país, como son los de Hernández et al. (2017), Buelvas et al. 2016 y los de Vargas y Bedoya (2015), ya que en todos la media es superior al valor intermedio de las escalas respectivas -de 1 a 5, o de 1 a 7 según el caso-.

Tabla 5. Media de la intención emprendedora en el total de estudiantes

\begin{tabular}{|l|c|}
\hline \multicolumn{2}{|c|}{ Intención emprendedora } \\
\hline Máximo & 5 \\
\hline Mínimo & 1 \\
\hline Media & 3,87 \\
\hline Desviación estándar & 1,04 \\
\hline Observaciones & 246 \\
\hline
\end{tabular}

Fuente Elaboración propia.

En la tabla 6 se muestra que para el global de estudiantes la calificación promedio de su percepción sobre la valoración que hace la sociedad sobre la creación de empresas sostenibles fue de $3.60^{4}$, lo que indica que en general es moderadamente buena, siendo los factores con mejor percepción la valoración positiva de la familia directa con 3.90 y vocación emprendedora sostenible en su región con 3.71, y la que cuenta con peor percepción es la favorabilidad positiva de la cultura general de país con 3.25 .
Al contrastar estos resultados con los de Vargas y Bedoya (2015) en estudiantes de nivel tecnológico en Colombia, se encuentra que para estos la media fue un poco más baja -3.55-, y como coincidencias que el factor mejor percibido es el apoyo de la familia, y que el peor es la favorabilidad de la cultura de sostenibilidad general de país, lo que corrobora que en las familias del sur occidente colombiano es bien vista la actividad empresarial en lo sostenible, pero que pueden haber factores que hacen que los estudiantes abordados no piensen lo mismo en cuanto a la generalidad del país.

\footnotetext{
${ }^{4}$ Promedio aritmético de la valoración general en el país $=3.25$, valoración por la familia $=3.90$, valoración por los amigos $=3.51$, valoración en la región = 3.71, valoración por los compañeros de estudio = 3.64.
} 
Tabla 6. Medias de los factores de valoración social

\begin{tabular}{|l|c|}
\hline \multicolumn{1}{|c|}{ Factores } & Total estudiantes \\
\hline Valoración general en el país & 3.25 \\
\hline Valoración por la familia & 3.90 \\
\hline Valoración por los amigos & 3.51 \\
\hline Valoración en la región & 3.71 \\
\hline Valoración por los compañeros de estudio & 3.64 \\
\hline Total valoración social & 3.60 \\
\hline
\end{tabular}

Fuente. Elaboración propia.

\subsection{Resultados correlacionales o inferenciales}

\subsubsection{Análisis de diferencias de medias.}

Al comparar por medio de la prueba T de Student, las medias de los factores de percepción social en los alumnos de contaduría frente a los de administración, se observa que en todos los casos son distintas, pero que estadísticamente ninguna de dichas diferencias es significativa ( $\mathrm{p}>0,05)$, por lo que en esta investigación no se puede concluir que la carrera profesional tenga efectos sobre la intención emprendedora de los estudiantes (Tabla 7).

Tabla 7. Medias de los factores de valoración social según carrera

\begin{tabular}{|l|c|c|c|}
\hline \multicolumn{1}{|c|}{ Factores } & $\begin{array}{c}\text { P valor Comparación } \\
\text { de medias }\end{array}$ & Administración & Contaduría \\
\hline Valoración general en el país & 0.887 & 3.24 & 3.26 \\
\hline Valoración por la familia & 0.404 & 3.86 & 3.96 \\
\hline Valoración por los amigos & 0.160 & 3.44 & 3.60 \\
\hline Valoración en la región & 0.323 & 3.66 & 3.78 \\
\hline $\begin{array}{l}\text { Valoración por los compañeros de } \\
\text { estudio }\end{array}$ & 0.990 & 3.64 & 3.64 \\
\hline Total valoración social & 0.296 & 3.57 & 3.65 \\
\hline
\end{tabular}

Fuente. Elaboración propia utilizando IBM SPSS Statistics 24.0.

A continuación, se presenta el modelo conceptual utilizado para revisar estadísticamente el efecto acumulado de los factores de percepción social sobre la intención emprendedora sostenible de los alumnos analizados, así como los resultados de dichas revisiones. 
5.2.2 Impacto de la valoración social en la intención emprendedora sostenible.

El siguiente es el modelo de regresión lineal utilizado para validar la hipótesis de este trabajo, es decir que cuanto mayor es en los estudiantes la percepción de una positiva valoración social respecto a la creación de empresas, mayor es su actitud emprendedora sostenible:

$$
\begin{gathered}
\text { Intención }=\beta_{0}+\beta_{1} \text { Valoraciónglobal } \\
+\epsilon
\end{gathered}
$$

Donde cada factor significa lo siguiente (Tabla 8):

Tabla 8. Significancia de cada factor

\begin{tabular}{|c|l|}
\hline Factor & \multicolumn{1}{|c|}{ Significado } \\
\hline Intención & Disposición actual de crear empresa. \\
\hline$\beta_{0}$ & Valor del intercepto de la recta de regresión con el eje Y. \\
\hline Valoración global & $\begin{array}{l}\text { Efecto acumulado de los factores de valoración social del } \\
\text { emprendimiento sostenible empresarial. }\end{array}$ \\
\hline$\beta_{1}$ & $\begin{array}{l}\text { Estimador de los parámetros que acompañan las variables y dan razón } \\
\text { del aumento o disminución de la intención emprendedora por cada } \\
\text { unidad de aumento de ellas. Valor de la pendiente. }\end{array}$ \\
\hline$\epsilon$ & Error aleatorio. \\
\hline
\end{tabular}

Fuente. Elaboración propia.

La tabla 9 muestra los resultados de la regresión lineal utilizada para determinar el impacto de la valoración total sobre la intención emprendedora sostenible de los alumnos abordados. Se aprecia que el valor de la $\mathrm{F}\left(6.598^{* *}\right)$ indica un buen ajuste general del modelo usado, que al ser el VIF (Factores de Inflación de la Varianza) 1.0 y el de prueba de Durwin Watson encontrarse por debajo de 1.66 , se deduce que en los datos utilizados no hay problema de multicolinealidad ni independencia. De igual manera que un R2 del 0.025 señala que la varianza de la intención emprendedora se explica en un $2.5 \%$ por la valoración social que se hace de ella.
La misma tabla señala que el beta es positivo y significativo a un $95 \%$ de confianza $\left(6.598^{* *}\right)$, lo que quiere decir que cada punto de aumento en la valoración total -sumatoria de la valoración en el país, en la familia, en los amigos, en la región y en los compañeros de estudiogenera un incremento de 0.22 puntos en el promedio de la intención emprendedora de los alumnos. Estos resultados que permiten probar la hipótesis de la presente investigación están en la línea de los hallazgos de Yang (2013), los de Vargas y Bedoya (2015), y los de Hernández et al. (2017). 
Tabla 9. Efecto de la valoración social total sobre la intención emprendedora

\begin{tabular}{|c|c|}
\hline Valoración social total & Valores estadísticos \\
\hline F & $6.598^{* *}$ \\
VIF & 1.0 \\
Durwin Watson \\
R2 Ajustado \\
$\beta$ \\
t \\
\hline
\end{tabular}

Fuente. Elaboración propia utilizando IBM SPSS Statistics 24.0.

\section{Conclusiones}

L as siguientes son las principales conclusiones de esta investigación que buscando aportar nuevos indicios sobre la relación de las intenciones emprendedoras y la creación de empresas en el ámbito universitario como factores de sostenibilidad social y económica para las regiones, abordó 246 estudiantes de contaduría pública y administración de empresas residentes en ciudades pequeñas y no principales de Colombia:

La intención emprendedora es alta en el país y la región abordada, esto se comprobó al encontrar que casi un $70 \%$ de los alumnos encuestados están dispuestos a ser empresarios, lo que coincidió con los resultados de otros estudios realizados a nivel nacional. Lo señalado es alentador, especialmente para la zona geográfica analizada que históricamente ha tenido múltiples problemas sociales, pues según las investigaciones y literatura previas, la creación de empresas influye positivamente en el avance económico y social, especialmente cuando estas nuevas organizaciones son manejadas con criterios de responsabilidad social empresarial.

En las ciudades colombianas tanto grandes como pequeñas, los alumnos tienen una positiva percepción general de la norma subjetiva o valoración de la sociedad respecto a la creación de empresas, y el apoyo de la familia se constituye en el factor que observan como más positivo al respecto.

La valoración social tiene un impacto positivo sobre la intención emprendedora universitaria regional, por esto se debería incrementar su permanente fomento dentro de los programas de desarrollo empresarial, lo cual podría apoyarse en campañas de divulgación masiva al interior y fuera de las universidades y de la presentación en los medios de comunicación de casos de empresarios exitosos y comprometidos con la sostenibilidad global. 
Como limitaciones del trabajo pueden señalarse que incluyó únicamente dos carreras de pregrado, pues haber abordado otros programas académicos, inclusive de posgrado, generaría un conocimiento más amplio de la intención emprendedora universitaria regional. Entre las posibles líneas de investigación futura que pueden derivarse de esta se plantean las siguientes: a) aplicar la encuesta en más carreras y en otras pequeñas ciudades de Colombia y de otros países, de tal manera que se produzca evidencia más fuerte sobre las características del emprendimiento regional; b) verificar cómo la formación en emprendimiento y desarrollo

\section{Referencias}

Ajzen, I. (1991). The theory of planned behavior. Organisational Behavior and Human Decision Processes, 50, 179-211.

Ajzen, I., y Fishbein, M. (1980). Understanding attitudes and predicting social behavior. Englewood Cliffs, NJ: Prentice Hall.

Alonso, P. (2012). La configuración de la intención emprendedora entre académicos responsables de proyectos de investigación en España. Un enfoque de género. (Tesis para optar por el título de doctor internacional). Santander, España: Facultad de Ciencias Económicas y Empresariales de la Universidad de Cantabria.

Audretsch, B., Belitski, M., y Desai, S. (2007). Entrepreneurship capital and economic growth. Oxford Review of Economic Policy, 23(1), 63-78.

Bae, T., Qian, S., Miao, C., y Fiet, J. (2014). The relationship between entrepreneurship education and entrepreneurial intentions: A meta-analytic review. Entrepreneurship theory and practice, 38(2), 217-225.

Buelvas, P., Franco, C., García, G., Gómez, L., López, S., Mátíz, F., Moreno, J., Osorio, F., Pereira, F., y Varela, R. (2017). Informe GEM: actividad empresarial colombiana 2016. Santiago de Cali, Colombia: Editorial Universidad ICESI. sostenible está influyendo sobre la intención emprendedora de los alumnos de la región estudiada y de las nuevas que se consideren estratégicas.

Los resultados de esta investigación son importantes para el sector gubernamental, el privado y para el académico, pues les ratifica que mejorando la percepción que tienen los alumnos sobre el entorno empresarial, es posible que se decidan con más frecuencia e intensidad por contribuir al desarrollo económico y social de sus regiones mediante la creación de empresas sostenibles y sustentables.
Chan, M. M., Escalante, M. N., y Robles, M. E. (2015). Comparabilidad en el nivel de emprendimiento de los alumnos de dos universidades: México y Puerto Rico. Revista Internacional Administración y Finanzas, 8(5), 1-14.

Cortés M., H., y Peña R., J. (2015). De la sostenibilidad a la sustentabilidad. Modelo de desarrollo sustentable para su implementación en políticas y proyectos. Rev. esc.adm.neg, (78), 40-55.

Diez, S. (2016). La actitud conductual en las intenciones emprendedoras. Revista Empresarial, ICE-FEE-UCSG, 10(2), 42-48.

Fernández, M. (2017). Estudio de las variables que afectan a la intención emprendedora en jóvenes universitarios. (Trabajo para optar por el título de doble grado en Finanzas y Contabilidad + Relaciones Laborales y Recursos Humanos). Universidad de Sevilla. Recuperado de https://bit.ly/2QuUGIc

García, Y., Mendoza, I., y Romo, L. (2017). Relación entre la intención de la elección de la carrera de Ingeniería Civil y la iniciativa emprendedora de los estudiantes de una institución de educación superior tecnológica en Hidalgo, México. European Scientific Journal, 13(34), 101-118. 
Gómez, S. (2010). Influencia de los factores institucionales sobre la actividad emprendedora corporativa. Un análisis causal. (Tesis para optar al título de doctorado). Universidad de Granada.

González, R., Torres, V., y Tinoco, M. (2017). Análisis empírico de los determinantes del emprendimiento en estudiantes universitarios. El caso de la Universidad de Colima en México. Economía y Sociedad, 21(36), 43-59.

González, D. F. (2004). Incidencia del marco institucional en la capacidad emprendedora de los jóvenes de Andalucía. (Tesis para optar al título de doctorado). Universidad de Sevilla. Recuperado de http://fondosdigitales. us.es/tesis/tesis/287/incidencia-del-marcoinstitucional-en-la-capacidad-emprendedora-delos-jovenes-empresarios-de-andalucia/

Goyanes, M. (2015). Apoyo estructural en la intención emprendedora de estudiantes de periodismo y comunicación audiovisual en España. El profesional de la información, 24(1), 55-61.

Hernández, R., Díaz, J., Fernández, A., Almodóvar, M., Díaz, Á., y Fernández, Y. (2017). GUESSS. Intenciones $y$ actividades emprendedoras de los estudiantes de la Universidad de Extremadura. Extremadura, España: Editorial Universidad de Extremadura.

Jaimes, M. F., Jaramillo, J. M., y Pérez, Ch. M. (2017). Factores que inciden en la intención emprendedora de estudiantes del Centro Universitario Tempascaltepec. Revista Venezolana de Gerencia, 22(78), 210-231.

Jany, C. J. (2009). Fundamentos de muestreo. Investigación Integral de Mercados. Bogotá: McGraw-Hill.

Joensuu, S., Varamäki, E., y Viljamaa, A. (2015). Beyond intentions-what makes a student start a firm? Education + Training, 57(8/9), 853-873.

Krueger, N. (2000). The cognitive infrastructure of opportunity emergence. Entrepreneurship: Theory \& Practice, 24(3), 5-23.

Laguía, A., Moriano, J., Molero, F., y Gámez, J. (2017). Validación del cuestionario de intención emprendedora en una muestra de estudiantes universitarios de Colombia. Universitas Psychologica, 16(1), 1-14.

Liñán, F. (2004). Intention-based models of entrepreneurship education. Piccolla Impresa/Small Business, 3, 11-35.
Liñán, F., Moriano, J., y Jaén, I. (2016). Individualism and entrepreneurship: Does the pattern depend on the social context? International Small Business Journal, 34(6), 760-776.

Marín, L., Rubio, A., Sánchez, M. (2015). El impacto del entorno del estudiante en sus intenciones de crear una empresa cuando finalice sus estudios. Lan Harremanak, 32(1), 504-526.

Martínez, G., y Campos, T. (2011). La intención emprendedora en estudiantes universitarios: el caso de la Universidad de Deusto. Boletín de Estudios Económicos, 69 (211), 151-172.

Mercado S, A., y Gil, P. R. (2012, septiembre-diciembre). Características psicométricas del cuestionario para la evaluación del síndrome de quemarse por el trabajo en maestros mexicanos. Revista de Educación, 359, 260-273 Recuperado de https://www.mecd. gob.es/dctm/revista-de-educacion/articulosre359/ re35913.pdf?documentId $=0901 \mathrm{e} 72 \mathrm{~b} 813 \mathrm{~d} 72 \mathrm{~d} 1$

Mora, P. R. (2011). Estudio de actitudes emprendedoras con profesionales que crearon empresa. Revista EAN, 71, 70-83.

Moriano, L. J., Trejo, E., y Palací, D. F. (2001). El perfil psicosocial del emprendedor un estudio desde la perspectiva de los valores. Revista de Psicología Social, 16(2), 229-243.

Novillo, E., Sarmiento, C., Ollague, J., y Ramón, D. (2017). Análisis de actitud emprendedora en estudiantes universitarios: una perspectiva de género. INNOVA Research Journal, 2(8), 56-63.

Pérez R., Zárate, T. R., Díaz, C. N., Martínez S. J., y Vargas, L. J. (Comp.). (2015). Memorias quinto congreso en gestión, emprendimiento e innovación: emprendimiento sostenible, 2013. Recuperado de http://repository. ean.edu.co/handle/10882/1588

Ramirez-Salazar, M.P., Perez-Uribe, R, y SalcedoPerez, C (2018). A triple helix model based on open collaborative innovation in colombia: a proposal for higher education institutions. En Nair, Suja R. and Saiz-Álvarez, J. M. (Eds.) Handbook of Research on Ethics, Entrepreneurship, and Governance in Higher Education. IGI Global.

Real Academia Española (RAE). (2017). Término de emprender. Recuperado de http://dle.rae. es/?id=Esip $2 \mathrm{Nv}$ 
Rifelly, D., y Martdianty, F. (2012). Student's entrepreneurial intentions by using Theory of Planned Behavior. The Case in Indonesia. The South East Asian Journal, 6(2), 100-143.

Rodríguez, D. (2016). Emprendimiento sostenible, significado y dimensiones. Revista Katharsis, (21), 419-448. Recuperado de http://revistas.iue.edu.co/ index.php/katharsis

Rueda, I., Sánchez, L., Blanco, B., y Fernández, A. (2013). Motivos y frenos al emprendimiento: ¿Varía su importancia según el género? Serie estudios del IUDE, 13125. Recuperado de Rueda, S. I., Fernández, L. A., y Herrero, C. Á. (2012). Estudiantes universitarios Cantabria y emprendimiento: investigaron determinantes psicológicos de la intención de creación de un negocio propio. Faedpyme International Review, 1(2), 9-15.

Sánchez, J. C., Lanero, A., y Yurrebaso, A. (2005). Variables determinantes de la intención emprendedora en el contexto universitario. Revista de psicología social aplicada. 15(1), 37-59.

Saravia, R. J. (2010). El problema del crecimiento sostenible, ¿cómo crecer sin destruirse? Revista EAN, (69), 136-147.

Schaltegger, S., y Wagner, M. (2011). Sustainable Entrepreneurship and Sustainability Innovation: Categories and Interactions. Business Strategy and the Environment, 237 (July 2010), 222-237. doi:10.1002/ bse. 682

Schumpeter, J. S. (1950). Capitalism, Socialism and Democracy. New York: Harper and Row Publisher.

Shapero, A. (1984). Entrepreneurship in Economic Development. In C. Far (Ed.), Shaping the local Economy. Washintong International City Management Association.

Timmons, J. A., y Spinelli, S. (2004). New Venture Creation: Entrepreneurship for the 21.st Century. Boston: McGraw-Hill Irwin.
Vainrub, R. (2009). Una guía para emprendedores: convertir sueños en realidades. México, D. F.: Pearson Education.

Valencia, A., Montoya, I., y Montoya, L, (2015). Factores explicativos de las intenciones emprendedoras en estudiantes universitarios. Espacios, 36(5), 1-15.

Valencia A., Montoya, I., y Montoya, A. (2016). Intención emprendedora en estudiantes universitarios: Un estudio bibliométrico. Intangible Capital, 12 (4), 881-922.

Varela, R. (2008) Innovación Empresarial, Arte y Ciencia en la Creación de Empresas. Bogotá: Pearson Educación.

Vargas, L., y Bedoya, M. T. (2015). Determinantes psicológicos de la intención de creación de empresas en los aprendices del Centro de Gestión Tecnológica de Servicios del SENA - Cali. Trabajo de grado para optar por el título de Magister en Administración de Empresas en la Universidad del Valle en Cali, Colombia.

Yang J. (2013). The theory of planned behavior and prediction of entrepreneurial intention among Chinese Undergraduates. Social Behavior and Personality, 41(3), 367-376. 